\title{
Potential Advantages of Conducting Short Duration Visits to the Martian Surface
}

\author{
Chel Stromgren and Felipe Escobar \\ Binera, Inc. \\ 77 S. Washington St. \\ Rockville, MD 20850 \\ 301-686-8571 / 301-579-5533 \\ c.stromgren@binera.com / \\ f.escobar@binera.com
}

\author{
William Cirillo, Raymond G. Merrill, \& \\ Kandyce Goodliff \\ NASA Langley Research Center \\ 1 North Dryden Street, MS 462 \\ Hampton, VA 23681 \\ 757-864-1938 / 757-864-2762 / 757-864-4969 \\ william.m.cirillo@nasa.gov / \\ raymond.g.merrill@nasa.gov / \\ kandyce.e.goodliff@nasa.gov
}

\begin{abstract}
Recent NASA concepts for human missions to Mars, including the Evolvable Mars Campaign and Design Reference Architecture 5.0, have focused on the conduct of missions with long duration stays on the Martian surface. The decision to focus on long duration missions (typically to a single site) is driven by a desire to increase the perceived sustainability of the human Mars campaign, predicated on the assumption that sustainability is best achieved by maximizing the level of activity on the surface, providing for continuous growth in operations, and promoting pioneering of Mars.
\end{abstract}

However, executing a series of long duration missions to a single site is not the only option for human exploration of Mars that has been proposed. Other architectures have been evaluated that focus on missions with short duration surface stays, with each mission visiting a separate site on the surface. This type of architecture is less efficient in that elements are not typically reused from one mission to the next but requires a far less complex surface architecture.

There are potentially valid arguments to be made that a short duration, multiple site approach could result in different types of advantages when compared to the long duration, single site approach to Mars exploration, particularly for initial human missions to Mars. These arguments revolve around four areas: Achieved Value, Risk Mitigation, Developmental Affordability, and Operational Affordability \& Flexibility.

The question of Achieved Value relates to the prioritization of goals for Martian exploration. As discussed, goals related to pioneering and expanding human presence are often referenced as justifications for the long duration approach. However, there are other competing goals, including science and exploration. While there is not a clear consensus among planetary scientists, many have argued that the value of being able to visit multiple sites could outweigh the value of continually visiting a single site.

Risk Mitigation is a major concern for initial human missions to Mars. There are a number of hazards related to operating on the Martian surface that are not well characterized. It may be desirable to conduct a series of short duration missions to better understand the nature of these risks prior to committing to a long duration mission.

Developmental Affordability relates to the ability of NASA and its partners to develop and deploy the proposed architecture. Any human missions to Mars will be among the most complex endeavors ever undertaken. The capabilities that must be developed to enable any human Mars missions are extremely challenging. The total design, development, test, and evaluation (DDT\&E) budget required to develop just the essential capabilities alone will be substantial. If additional surface capabilities are required to support long duration surface stays, the development effort could be unaffordable.

Operational Affordability \& Flexibility relates to the continued costs to execute the Mars campaign. Long duration missions, even with some amount of in-situ resource utilization, require a significant level of resupply for every mission. This requires additional launches and in-space transportation assets, increasing the operational complexity and total operational cost.

This paper will explore each of the four potential advantages of short duration missions in detail. The authors will present comparisons between proposed long duration and short duration architectures through an evaluation of relevant performance, cost, and risk metrics.

\section{TABLE OF ConTents}

1. INTRODUCTION ...........................................................1

2. ASSESSMENT …...............................................................4

3. ACHIEVED VALUE ...............................................4

4. RISK MITIGATION.......................................................6

5. DEVELOPMENTAL AFFORDABILITY .......................8

6. OPERATIONAL COST \& FLEXIBILITY ...................10

7. INITIAL SHORT DURATION MISSIONS ..................11

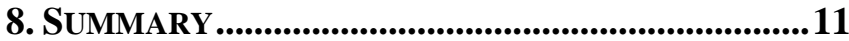

REFERENCES .........................................................13

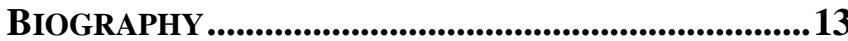

\section{INTRODUCTION}

The Apollo lunar landings are widely considered to be one of NASA's and humankind's greatest technological achievements. However, during the first lunar landing, Neil Armstrong and Buzz Aldrin spent only 22 hours on the lunar surface. The longest duration on the surface for any Apollo mission was 75 hours - just over three days. While early Apollo planning included concepts for extended exploration, such as long duration stays, habitats, and pressurized rovers [1], budgetary and political realities ultimately limited the program to only short duration sortietype missions. While the Apollo program achieved its stated objectives, many space enthusiasts and historians lament the fact that the extended concepts were never realized and that 
the lunar program was terminated after Apollo 17, with some blaming the lack of sustainability on the limited scope of the missions [2].

Now, almost 50 years later, NASA is beginning to actively plan for a human Mars campaign. Recently there has been significant progress in efforts to evaluate different architectural options to enable these missions. NASA's most recent plans include human visits to Mars orbit in the early 2030s and human visits to the surface of Mars in the late 2030s. Recent design activities, such as Design Reference Architecture 5.0 (DRA 5.0) [3] and particularly the Evolvable Mars Campaign (EMC) [4], are informing the planning and development of the human Mars architecture. As part of these activities, many of the same issues that were addressed in the Apollo program are also being evaluated in reference to a human Mars campaign. A primary area of interest is the content of the surface architecture and the level of activities conducted on Mars.

Both recent NASA design activities, Mars DRA 5.0 and the EMC, focus on missions with long-duration surface stays on Mars, with extensive surface infrastructure to support the crew and to enable exploration. The decision to focus on long duration missions is based on a desire to maximize the relative "value" achieved on the missions relative to the expected costs and risks of executing the Mars campaign. Essentially the argument that is being made is, "You are going all the way to Mars, you should maximize what you do once you get there." More specifically, if you are sending humans to the surface of Mars, you want to have them on the planet exploring for the entire duration of their stay at Mars.

On the surface this appears to be a valid argument. The cost of a human Mars campaign will likely exceed that for any other previous space program. The Mars program will therefore receive a huge amount of public attention and scrutiny. By maximizing the level of achievements, the program is therefore more likely to be initially funded and to continue to receive public and political support over time.

However, this argument oversimplifies the budgetary and political realities of executing a human Mars program. Architecting a sustainable Mars campaign is not simply a

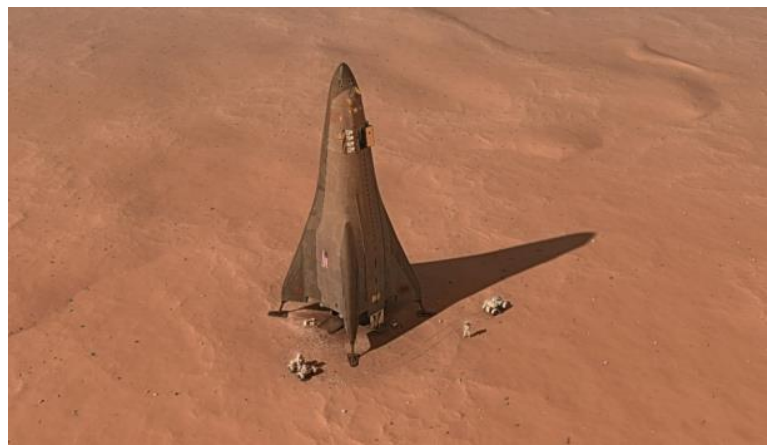

Figure I.1 - Lockheed Martin Lander for Short Duration Surface Missions (reprinted with permission from Lockheed Martin) process of maximizing the cost-benefit ratio, but rather it must establish a balance between what we want to achieve with what we can afford to achieve. The cost of providing the infrastructure required to enable long duration surface stays may require substantial added investments.

\section{Short Duration Surface Missions Versus Long Duration Surface Missions}

The capabilities required to deliver a crew to Mars vicinity and then to the surface are extensive. Supporting the crew for long durations on the Mars surface (typically 300-500 days) requires a significant amount of surface infrastructure and a large supply of logistics. Infrastructure requirements include a large habitat or habitats with closed-loop Environmental Control and Life Support System (ECLSS), a power system capable of providing power to the entire outpost during nighttime periods, unloading and transfer systems that allow cargo to be offloaded from multiple landers and moved to the outpost, and Extravehicular Activity (EVA) systems that allow the crew to operate on the surface. This is in addition to any other systems that are required to let the crew utilize their time on the surface, such as mobility systems to allow astronauts to explore the area around the outpost, science laboratories, and science packages.

The alternative to a long duration mission is a much simpler, short duration, "sortie" type mission. In this approach the crew (sometimes with as few as two astronauts) descend to the surface and spend a limited amount of time exploring the site, deploying scientific instruments and collecting samples, before returning to the Deep Space Transport (DST) spacecraft in orbit around Mars. The proposed surface duration of the missions varies but is typically from 7 days to 60 days. The missions are characterized by the use of a minimal amount of surface infrastructure and logistics. Under proposed short duration scenarios, the crew can either live in the Mars Ascent Vehicle (MAV) cabin or a minimal habitat (perhaps a copy of the MAV cabin) with open-loop ECLSS. Power generation and storage capability requirements are limited, as the supported infrastructure and power loads are much lower and the missions can be designed to avoid the longest night durations. These short duration missions do not have

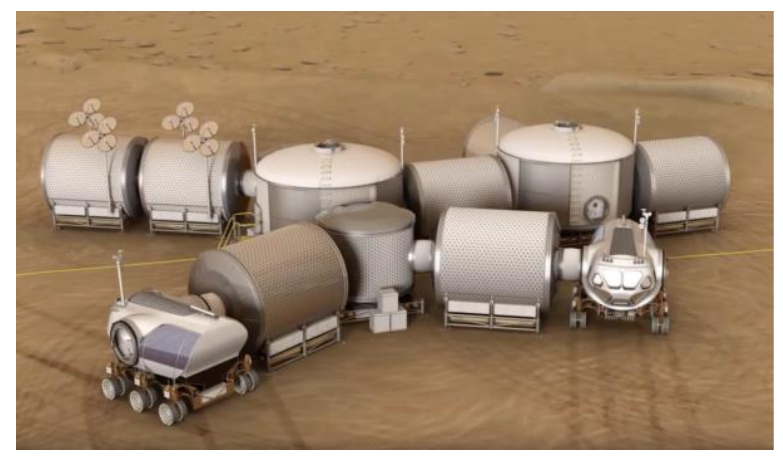

Figure I.2 - EMC Mars Outpost for Long Duration Surface Missions (build-up after multiple missions) 
to be copies of the very minimalistic approach employed in Apollo. With a limited amount of additional surface infrastructure (consisting of a small habitat, airlock, power system, and logistics storage) these missions could extend up to 60 days in duration and provide for a significant amount of exploration in that time.

The potential advantages of the short surface duration mission stem primarily from the greatly reduced complexity of the surface architecture and operations. With less required capability development, less element acquisition, and lower required deliveries to the Mars surface, the level of effort for development and operations can be reduced, therefore reducing the potential cost and risk of the campaign. Illustrations of the surface elements for representative short surface duration and long surface duration are pictured in Figure I.1 [5] and Figure I.2 [6].

\section{Rationale for Long Duration Surface Missions}

Recent design exercises have focused on long duration surface missions. For both EMC and DRA 5.0, this decision was made in an attempt to maximize the level of achievements and value accomplished during the Mars campaign, relative to the cost and risk. Both efforts reference established Mars Exploration Program Analysis Group (MEPAG) goals and objectives [7] (discussed further in Section III.E) but offer slightly different rationale for selecting the long duration approach. Each study also varied in the architectural solution selected.

For EMC, the choice was made to focus on long-duration surface stays to a single outpost site, reusing capabilities on successive missions, conducting crewed missions on every other Mars departure opportunity. This choice was adopted based on a stated goal to promote pioneering and to expand human presence. The mission architects link the achievement of these goals to specific objectives that include: maximizing the time that humans spend on Mars, preparing to live off the land, establishing a path to permanent human presence, and increasing the tempo of capabilities and activities [8]. The decision to focus on build-up of capabilities at a single site was based on this emphasis on pioneering and on a desire to promote affordability and increase sustainability through the reuse of elements.

For DRA 5.0, the campaign includes three long duration surface missions to three different locations on the Martian surface, conducting crewed missions on every Mars departure opportunity. DRA 5.0 architects performed an extensive analysis of Mars exploration goals and objectives and selected the long duration, multiple site approach as providing the greatest overall value, balancing both science achievements and expanding human presence [9]. It should be noted that the approach adopted in DRA 5.0, deploying a long duration surface infrastructure to a new location at every Mars opportunity, requires a very high launch rate, with the crewed elements for one mission being deployed at the same time as cargo elements for the next mission. DRA
5.0 also requires the acquisition of extensive exploration hardware, potentially presenting challenges to affordability and sustainability.

\section{Single Site Versus Multiple Site}

Surface stay time is not the only discriminator in determining mission complexity. The other major factor is the number of surface sites visited during the campaign whether successive missions return to the same site and reuse infrastructure or visit new sites on each successive mission. Short duration missions are insensitive to the number of sites visited because the surface elements are relatively simple and are not generally reusable.

For long duration missions, the number of sites can be a major factor in complexity and cost. If missions return to the same site, major pieces of infrastructure such as the habitat, power generation, and rovers can be reused, eliminating the need to reacquire and redeliver those elements. This can reduce the complexity and cost of subsequent missions.

Long duration missions to multiple sites are the least affordable option. All required infrastructure elements must be flown for all missions, significantly increasing the number of required landers, the in-space propulsion stages, and the number of launch vehicles.

\section{Short Surface Duration Versus Short-Stay Mars Missions}

It is important to distinguish between the concept of a short duration surface mission and a short-stay Mars mission. These terms are somewhat ambiguous and can result in some misunderstanding when comparing different solutions.

In this paper the terms 'short duration' and 'long duration' are used to identify the amount of time that the crew spends on the surface of Mars. This parameter is independent of but related to another factor, which is the amount of time that the crew spends in Mars vicinity, whether on the surface or in orbit. This parameter has historically been referred to as the 'Mars Stay Time'.

Missions with a short Mars stay time, or 'short-stay' missions, utilize an opposition class trajectory for transit between Earth and Mars. These missions typically have a total in-space duration of 500-900 days, depending on the opportunity and the type of propulsion utilized, and a stay time at Mars of between 30 and 90 days. These missions are characterized by shorter overall mission durations and significantly higher delta $\mathrm{V}$ and thermal requirements.

Missions with a long Mars stay time, or 'Long-stay' missions, utilize a conjunction class trajectory, where minimal energy transits are utilized for transfer between Earth and Mars. These missions typically have total durations of approx. 1000-1100 days with stay times between 350-500 days at Mars, depending on the type of propulsion. 
Because of the short overall times at Mars, short-stay opposition class missions can only enable short duration surface visits. For long-stay missions, the crew can complete either short duration or long duration surface stays. If a short surface duration visit is conducted as part of a long-stay, conjunction class mission, the crew must spend the remainder of the overall Mars stay time in the DST, in orbit around Mars.

Many architecture studies of short surface duration versus long surface duration missions concentrate on comparing the 'long stay, conjunction class' architecture with the 'short stay, opposition class' architecture, including the differences in propulsive requirements and total mission duration. The analysis presented in this paper assumes that both the short surface duration and the long surface duration missions would be conducted as part of a long-stay, conjunction class architecture. This assumption reflects current NASA planning for the in-space propulsion architecture for Mars missions and allows the analysis to focus on a comparison on differences in the surface approach, rather than concentrating on differences in the in-space architecture. In addition, the long-stay architecture would support an eventual transition from short surface duration missions to long surface duration missions, if desired.

\section{ASSESSMent}

In this paper, the authors identify and explore four distinct areas where short duration surface missions may present certain advantages over long duration missions. These four areas are:

$\begin{array}{ll}\circ & \text { Achieved Exploration Value } \\ \circ & \text { Risk Mitigation } \\ \circ & \text { Developmental Affordability } \\ \circ & \text { Operational Affordability \& Flexibility }\end{array}$

This paper will compare campaign options that focus on a short duration, multiple site campaign to the long duration single site campaign that is being investigated as the primary option in NASA's Evolvable Mars Campaign. Cost, risk, and performance data integrated from various studies are used to evaluate the two options and make recommendations for further research.

\section{AChieved VAlue}

The two most recent NASA human mars mission design activities have focused on a long surface duration approach to surface exploration. In both cases the selection of a long duration approach was related to a desire to maximize the achieved value of the campaign, relative to the cost and risk. Campaign value is measured in relation to the achievement of an established set of goals and objectives. The most comprehensive set of goals and objectives for human Mars exploration was established by the MEPAG [7]. It is worth examining these goals and objectives in detail and evaluating what can be accomplished on a series of short duration surface mission versus long stay missions.

\section{MEPAG Goals and Objectives}

For both of the architecture studies rationale for the decision to focus on long duration architectures was related to a desire to maximize the value achieved by the campaign to the established Mars exploration goals of 'Science' and 'Expanding Human Presence'. These two sets of goals, which were established by the MEPAG and are detailed within MEPAG documentation, serve as the basis for informing what types of exploration activities could be achieved during a set of human Mars missions.

The objectives within MEPAG Goals I-III concentrate on scientific discoveries at Mars. These objectives relate to the search for life, climate science, and geology/geophysics. The objectives within MEPAG Goal IV are related to Expanding Human Presence on Mars. These Goal IV objectives involve activities designed to gather knowledge about Mars, related to the operational environment and potential resources, to testing exploration systems and operational capabilities, and to enabling future missions.

The time that the crews spend on the surface, the number of sites that they visit, the level of infrastructure deployed, and the reuse of infrastructure all contribute to the determination of how each of the MEPAG goals and objectives are satisfied. The rationale for why a specific architecture was selected for each study is similar for both DRA 5.0 and EMC, although the specific arguments and the architecture selected differ considerably.

\section{DRA 5.0}

As part of the DRA 5.0 effort, the analysis team conducted a thorough study of the sometimes competing, sometimes complementary goals. Evaluating the objectives and specific activities related to those objectives, the team evaluated four potential mission approaches:
- Short Duration Single Site
- Short Duration Multiple Site
- Long Duration Single Site
○ Long Duration Multiple Site

The team then separately evaluated the relative value of each of the four approaches in the two general areas of benefit: MEPAG I-III Science and MEPAG IV Expanding Human Presence. Results from the DRA 5.0 analysis are shown in Figure III.1 [9]. 

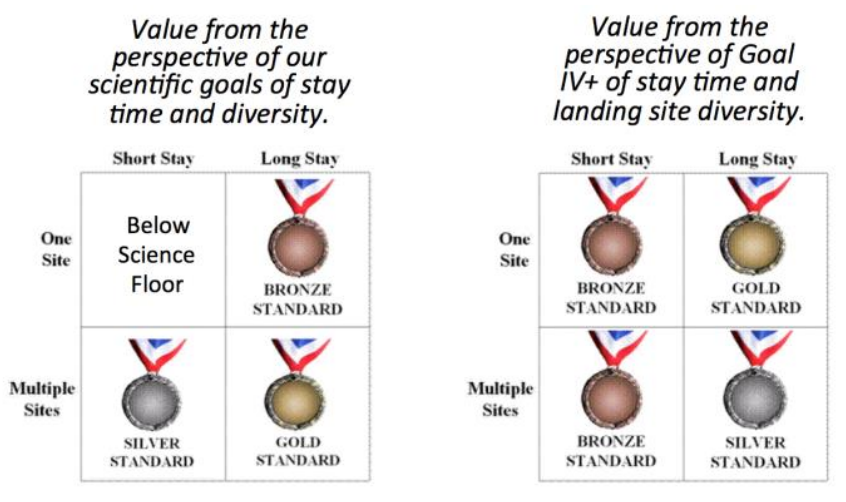

Figure III.1 - DRA 5.0 Goals Assessment

In the areas of MEPAG I-III Science, the analysis team determined that the most productive mission type would be long durations at multiple sites. The team rated this as the "Gold" standard. Short durations at multiple sites was rated as the "Silver" standard and long duration, single site as "Bronze". The team also determined that short duration single-site missions were below the acceptable standard for science. In their discussion the team specifically recommended that missions be conducted to multiple sites as part of a comprehensive science program and that even with extended mobility a single site campaign would not be able to satisfy all science goals.

For MEPAG IV Expanding Human Presence, the analysis team determined that the long duration, single site approach was the "gold" standard, allowing for an expanded human presence and long-term testing of capabilities. The long duration, multiple-site was rated as "silver" and all short duration missions, at a single site or multiple sites as "bronze".

DRA 5.0 summarized the somewhat conflicting results of the two assessments as follows, "To use human explorers effectively in addressing these scientific questions, the first three human missions to Mars should be to three different geographic sites. The Goal IV objectives lend themselves best to repeated visits to a specific site on Mars, however." Ultimately the team selected a long duration, multiple site approach as having the best balance between MEPAG goals.

\section{EMC}

The EMC specified return to a single-site as a ground rule and assumption for development of the architecture. The desire to reuse surface infrastructure was determined to be a primary goal of the campaign. In addition, EMC explicitly prioritized pioneering and extended human presence as a primary goal of the Mars campaign [8]. Based on this prioritization, the EMC selected a single-site, long duration architecture as the primary option for investigation.

EMC options all include a build-up of infrastructure at the single surface location for the purpose of enabling multiple, successive missions. Utilizing a single surface site increases the efficiency of conducting long duration missions, allowing surface elements to be reused, with each successive mission adding to the infrastructure. Reuse of elements at a single site also facilitates the delivery of advanced capabilities such as ISRU propellant production and nuclear power, which increases the self-reliance of each mission, thereby reducing resupply requirements from Earth. The EMC enhances the conduct of Science goals at the single site through the extensive application of mobility and EVAs.

EMC did not specifically compare the relative value of visiting multiple sites versus a single site, due to the single site ground rule. EMC did evaluate short duration single site options as alternatives, primarily to enhance affordability. These short surface stay options were limited to only the initial mission(s), with an immediate transition to long duration missions by the second or third crewed mission. However, EMC retained the long surface duration missions as the primary option for analysis.

\section{Achieved Value Assessment}

There is little doubt that in an environment that was not constrained by affordability or operability issues the 'long duration, multiple site' approach, as adopted in DRA 5.0, would yield the greatest possible value in relation to achieving MEPAG goals and objectives. However, it could be difficult to implement that type of architecture under reasonably anticipated budget and launch constraints. The anticipated SLS launch rate would likely not support such an architecture without unreasonably long periods between crewed missions. It is also probable that such an architecture would not be affordable, due the number of elements and launch vehicles that must be acquired to support each crewed mission. Similarly, the 'short duration, single site' architecture is not considered to be a reasonable alternative. As concluded in DRA 5.0, that option does not achieve satisfactory performance against either MEPAG goal area. Therefore it is productive to limit the discussion to either:

\section{- 'Long duration, single site' missions, or}

○ 'Short duration, multiple site' missions

Comparing the achieved value between these two options depends heavily on how different, sometimes competing, sometimes complimentary, goals and objectives are both interpreted and prioritized.

Return on 'Science' Goals and Objectives-The DRA 5.0 analysis indicates that a short duration, multiple site campaign provides a greater scientific return related to MEPAG Goals I-III than any single site approach. The variability of terrain and conditions on Mars virtually requires different landing sites in order to complete any sort of comprehensive scientific survey. Although most long duration missions anticipate extended mobility via pressurized rovers, the limit of these excursions will be only of few hundred kilometers or less. This is simply not enough range from a single site to capture the variability in Mars terrain. Single sites will always be limited in the types 
of terrain that astronauts can explore and collect samples from. In addition, the extra time available to conduct science offered by a long duration mission may not be as beneficial as anticipated. The amount of material that can be returned to Earth is still limited by the MAV capabilities. So, any additional samples collected would have to be evaluated by the astronauts on the surface. Based on the defined goals and objectives, it appears that a campaign that allows astronauts to visit multiple sites on the surface will provide greater scientific return than a campaign that visits only a single site.

Return on 'Expanding Human Presence Goals and Objectives' - The arguments in favor of long duration made for both DRA 5.0 and EMC assume that immediate build-up of duration and capabilities is the optimal way to complete the MEPAG IV objectives and to enhance human presence. In some ways this misstates the intent of the MEPAG goals and objectives as written. The MEPAG IV goal was intended to define exploration objectives that would "prepare for a sustained human presence" [7], not necessarily accomplish that expanded presence directly.

While a long duration Martian outpost is undoubtedly the most effective mechanism to accomplish some of these goals, there are others, such as discovering resources that are better supported through visiting different sites. In addition, the MEPAG IV objectives specifically refer to "developing reliable and robust exploration systems" as an objective. As discussed further in the Risk Mitigation section of this paper, a progressive exploration campaign that limits initial exposure to risk and grows capabilities over time may be the best approach to achieving this objective.

While a long duration, single site surface approach may maximize the value achieved in certain areas related to expanding human presence, there are other areas where a short duration, multiple site approach may equal or exceed the value achieved.

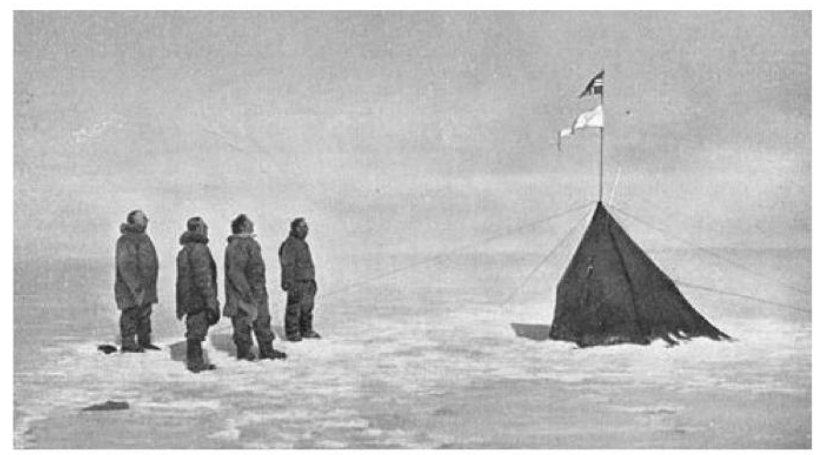

Figure IV.1 - $1^{\text {st }}$ Amundsen South Pole Expedition (Credit Amundsen, Roald: The South Pole, Vol. II, first published by John Murray, London 1913. Photo facing page 134)

\section{Risk Mitigation}

Human exploration of the Mars surface will expose the astronauts and architecture elements to types of risks that have never before been encountered. It is critical to the success of the campaign that these risks be addressed in a systematic manner that progressively exposes the missions and crews to these risks, characterizes the nature of the risks and how to mitigate them in an effective manner.

Proponents of the long duration Mars mission often compare their ambitions to the achievements made in the field of Antarctic exploration [10]. These individuals foresee the ideal approach to Mars as being akin to the deployment of Antarctic research stations such as McMurdo Station on Ross Island or Amundsen-Scott Station at the South Pole. Both of these facilities support extended human occupancy in a very hostile environment, enabling science and exploration. However, it should be realized that these facilities did not come into existence during the initial exploration of Antarctica. Roald Amundsen first reached the South Pole in 1911 after an 18-month journey, as illustrated in Figure IV.1. He and his crew stayed only a few days at the South Pole. Subsequent expeditions to the Antarctic progressively improved knowledge about the environment. Amundsen-Scott station was not established until 1956, 45 years after the initial visit, illustrated in Figure IV.2.

The Antarctic experience actually demonstrates a more measured, more realistic approach to exploration. Because of the severity of the environment and the inherent risks, it would have been logistically, financially, and politically difficult to immediately establish any sort of semipermanent base at the South Pole. Rather, explorers spent 45 years exploring the Antarctic environment. Only then were they able to transition to a near-permanent presence.

This is an approach that applies directly to the exploration of Mars. Despite the numerous robotic missions that have been conducted in orbit and to the surface, our knowledge of conditions and threats on the Mars surface and how they will impact human missions is extremely limited. As such, there are a number of significant risks to the Mars surface

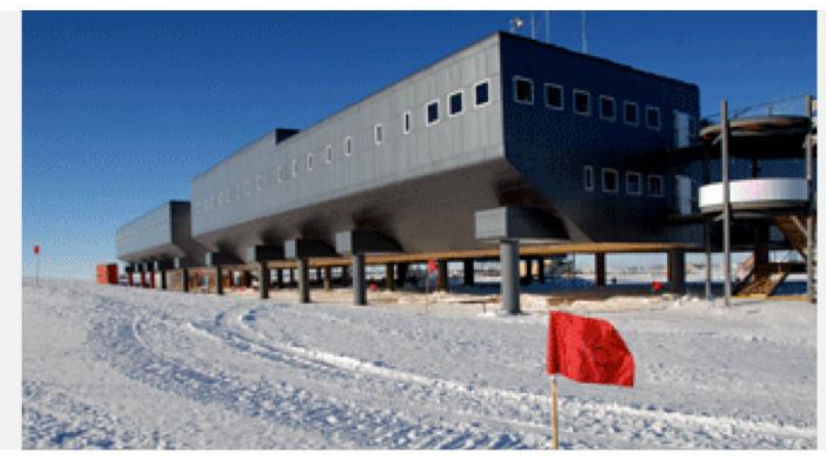

Figure IV.2 - Amundsen-Scott Research Station

(Credit Scot Jackson, National Science Foundation) 
mission and Mars astronauts.

\section{Safety Risk on the Martian Surface}

Surface risks are extensively described in the National Research Council Report "Safe on Mars" [11]. These risks include:

Chemical Environmental Hazards on Systems - The impact of soil and airborne dust and perchlorates on the safe operation of critical systems is a major threat to the mission and the crew [12]. Degradation of equipment, including corrosion, could lead to critical impairment of life support or transportation functionality. The more complex the surface systems are, and the longer those systems are exposed, the greater the risk posed to the mission and the crew. This is particularly significant for architectures such as those presented in the EMC, where surface infrastructure is planned for reuse on multiple successive missions. In that case, elements such as the surface habitat and fission power units are to be used for up to 15 years.

Surface impacts on the MAV are of particular concern. The MAV is a critical element to crew safety. The longer the MAV is on the surface, the greater the exposure to surface conditions and the greater the risk. In the proposed EMC architecture, the MAV is on the surface for nearly two years prior to ascent due to the length of the surface mission and the need to generate ISRU propellant prior to crew arrival.

Chemical Environmental Hazards on Crew-The presence of toxic constituents, including perchlorates, in soil and airborne dust could present a risk to the crew [12]. The presence of these toxins and their impact on the crew has not been adequately defined and may not be prior to the first human missions. Longer duration missions result in greater exposure to the crew.

Hazards from Atmospheric Dynamics-Although atmospheric electrical activity on Mars has not been observed to the same degree as lightning generated by charged ice particles on Earth, there is evidence that electrical activity can be generated by Martian dust storms and dust devils. A potentially greater risk to crew and system safety is the lack of local electrical ground on Mars which significantly increases the potential for electrostatic discharge, specifically during EVA/Rover operations that can result in charge buildup due to surface friction. The severity and potential impacts of atmospheric dynamics on exploration systems from electrostatic discharge has not been fully investigated.

Physical Environmental Hazards-Despite experience with robotic landers, there is still uncertainty in the physical makeup of the Martian surface and the variability of that makeup. There are hazards related to the physical interaction of exploration infrastructure with surface, including tip-over, abrasion, and impact, particularly in complex terrain environments where the impact of surface winds are less understood. More complex architectures, which require unloading of elements from landers, transportation across the surface, and integration are significantly more susceptible to these hazards.

An initial long-stay mission would result in full exposure to each of these risks with limited opportunities to better understand the nature of the risks and potential mitigation solutions prior to the crewed mission. Initial short duration missions present a significantly more conservative approach to mitigating these surface risks. Short duration missions with minimal infrastructure would result in less exposure to each of the risks and the conduct of those missions would allow for an evaluation of the surface impacts on systems with lower initial durations of exposure and/or lower criticality. This knowledge can then be used to improve the performance and safety of future systems.

\section{Other Mars Surface Risks}

In addition to the risks described above there are other evaluations and surveys that, while they may or may not directly impact the safety of initial missions, could be important to complete before selecting a site for a permanent Mars outpost. A key element to eventually making long duration surface stays sustainable is lessening the need for resupply from Earth. This means that local production of logistics, such as propellant, gas, water, and food, must be explored and the resources and processes required to enable must be established.

Availability of Resources-Water and other resources, extracted from Martian atmosphere and regolith for the production of power and propulsion consumables, will be important in allowing a Mars outpost to reduce dependence on Earth and expand human presence. Selecting a site with abundant available resources will be critical. Exploration of multiple sites in different geographical and geological areas will allow for a better understanding of resource availability prior to selecting an outpost site [9].

Demonstration of Resource Processing-Demonstrating the capability to collect and process in-situ resources will also be critical. ISRU systems will be complex and have to operate for long-periods in a hostile environment. It will be desirable to improve the operability and reliability of these types of systems, prior to putting them in the critical path for supporting the crew of producing propellant for the MAV. ISRU demonstration activities will be completed as part of the Mars 2020 Mars Oxygen ISRU Experiment (MOXIE). Additional demonstrations could be completed as part of short duration missions.

\section{Alternate Risks of Short Duration Surface Missions}

Proponents of the long duration mission point out that there are other threats where the risk of the short duration mission could be greater than for the long duration. Typically, a discussion of the following risks is used as a justification for the long duration surface missions. 
In-Space Health Risk to Crew-If, as assumed in the analysis presented in this paper, that short surface duration missions are conducted as part of a long-stay conjunction class mission, then the crew would have to spend the remainder (the time not spent on the surface) of the Mars vicinity time in the spacecraft in orbit around Mars. This increases the total exposure to known risks to crew health from radiation and microgravity.

While the risks to the crew in space are significant, the total time spent in space for the short duration is only moderately longer than for the long duration. For the proposed EMC architecture, with a 350-day stay in Mars vicinity, the crew on a long duration would spend approximately 875 days in space. For the short surface duration mission, assuming a 30-day surface stay, the crew would spend about 1,170 days in space, an increase of approximately $33 \%$.

In addition, the risk associated with long in-space durations is an area NASA must address in order to proceed with the current exploration plan. The planned initial 2033 orbital mission will keep the crew in space for the entire 1100 day Orbital mission. The risk of a short duration mission will be no greater than for that mission. In addition, even if NASA decides not to proceed with an orbital mission, the crew must still be able to spend the entire Mars vicinity duration in space. There is always the chance that the crew may be unable to descend to the surface or will need to abort to orbit from the surface due to a technical failure or other hazard. Additional research is required to determine the magnitude of risk to human health of long durations in space and whether NASA will be able to sufficiently mitigate those risks to enable missions of different durations.

Crew Acclimatization-One major issue with short surface stay missions is the amount of time that the crew will be able to dedicate to exploration versus simply acclimating to the Mars surface environment. After a nearly one year journey from Earth to Mars, the crew will have some level of deconditioning due to the micro-gravity in-space environment. There will be some period required for the crew to gain functionality in the Mars gravity environment (even at $1 / 6 \mathrm{~g}$ ). If this period is extensive, it could significantly reduce the productivity of a short stay mission. In addition, if the crew tries to do too much on the surface prior to acclimatization, there is a risk of injury and negative health impact.

Increased Criticality of Surface Elements-One advantage of an extensive surface architecture is that it provides redundancy and increased safety for the crew. If there is a failure in habitation capabilities, the crew can shelter in other elements on the surface until the problem is resolved or until they can abort. Similarly, if there are issues with the MAV, the crew can shelter for extensive periods on the surface until the issues are resolved.

There is currently no definitive answer as to what type of mission, short surface stay or long surface stay, would result in the greatest risk to the crew or to how those risks can be reduced. As NASA proceeds with architecting the Mars mission it will be important to conduct further research and a quantitative risk assessment to understand and compare these risks. However, there is a possibility that the less complex infrastructure and shorter exposure times offered by the short surface stay mission could reduce overall risk.

\section{DeVelopmental AfFordability}

The most significant potential advantage of conducting initial short duration missions to Mars is in the area of affordability. A human Mars campaign will be one of the most challenging, and most expensive, activities ever attempted. It is vital that an approach be selected that allows costs to be controlled and spread out in a way that makes the undertaking affordable. It is unlikely that NASA will receive a substantial increase in budget, or any increase at all, to support the human Mars campaign. It is therefore critical that activities are structured in such a way that the associated costs can be spread out to maintain a reasonable budget. Even with new acquisition methods and international partner participation it is likely that affordability will remain challenging.

Any human mission to Mars will require the development of a new and complex set of capabilities. Required capabilities include crew and cargo launch systems, an in-space habitation system to safely carry the crew to and from Mars, a propulsion system(s) to transport the crew and cargo, ECLSS to keep the crew alive, communication systems to reach back to Earth, and EVA systems to allow the crew to operate out of the spacecraft.

Reaching the surface requires an additional set of capabilities. An entry, descent, and landing (EDL) system capable of delivering $10 \mathrm{~s}$ of metric tons of cargo to the surface with a high level of accuracy and safety is required to support a human mission. An ascent vehicle that can be delivered on that lander and which can be launched from the surface to return the crew safely to the DST is also necessary. Finally, any capabilities that are required for the

Table V.1 - DDT\&E Cost Estimates for Human Mars Mission Architecture, By Phase, Corrected to FY2017 Dollars - Price et al (2009)

\begin{tabular}{|l|r|l|r|l|r|}
\hline \multicolumn{2}{|c|}{ In-Space Transportation } & \multicolumn{2}{c|}{ Surface Access and Ascent } & \multicolumn{2}{c|}{ Surface Infrastructure } \\
\hline Capability & FY17 Cost & Capability & FY17 Cost & Capability & FY17 Cost \\
\hline In-Space Propulsion & \$4.62B & Descent/Ascent Vehicle & \$17.44B & Surface Habitat & \$8.09B \\
\hline Deep Space Habitat & $\$ 6.33 \mathrm{~B}$ & & & Power/Logistics & \$6.50B \\
\hline Total & \$10.94B & Total & \$17.44B & Total & \$14.59B \\
\hline
\end{tabular}


crew to live and operate on the surface must also be developed.

NASA's current approach to achieving affordability in the Mars campaign is based on a successive increase in the goals of the program and in the capabilities that must be developed to achieve those goals. The current exploration plan envisions a staged approach to Mars exploration. Initially, the technologies, capabilities, and knowledge required for human deep space missions will be developed on Earth, at the ISS, and in cis-lunar space. Next, the transportation and in-space habitation capabilities required for the Mars mission will be developed and tested, culminating with a human Mars orbital mission in the early 2030s. Then, the capabilities required for the surface mission will be developed, with the goal of landing humans on the surface in the late 2030s.

The content of each of the stages must be carefully managed to limit required capability development and cost. In Price et al (2009) [13], the Aerospace Corporation completed a set of cost estimates for the development of required Mars exploration elements. While actual costs will depend upon a number of factors, including specific technologies and architecture, acquisition method, and partnerships, these costs can serve as a measure of the relative level of effort (LOE) required to develop different capabilities. The costs used in this paper generally agree with those described by the National Research Council (2014) [14,15]. Estimated DDT\&E costs, segregated by stage, are summarized in Table V.1.

Price et al. estimate the cost for DDT\&E of the in-space transportation system, including the deep space habitat and the in-space propulsion stages, to be FY17\$10.9B (corrected from FY2009 dollars), as shown in Table V.1. Developments for this stage would also include ECLSS, EVA, medical, autonomy, and other required investments.

Costs for the development of capabilities to enable a short duration surface mission are dominated by the cost of the Mars Lander and Mars Ascent Vehicle. Other required capabilities would include surface EVA and potentially mobility systems. Developmental costs for aerobraking and aerocapture are also included in this phase, if applicable. It is assumed that short duration missions do not require the development of extensive surface habitation capabilities. Crew can either live in the MAV cabin or a minimal habitat (perhaps a copy of the MAV cabin) and open-loop ECLSS can be utilized. Power generation and storage capabilities are also limited. Price et al estimate the cost of these capabilities to be FY17\$17.4B.

The capabilities required to then enable a long duration surface mission are substantial. These capabilities include: a long duration habitat, with closed-loop ECLSS and extensive environmental monitoring, mobility, lander offloading and cargo transport, and logistics delivery systems. In addition, a power generation and storage system capable of supporting the outpost, including nighttime periods, is required. Price et al estimate the total cost of these capabilities to be FY17\$14.6B.

The cost estimate for the extended surface infrastructure is likely conservative. Price at al assumed a radioisotope power system, as opposed to a fission surface power system (FSPS), which is anticipated in both DRA 5.0 and the EMC. FSPS will likely have a significantly higher cost to develop. In addition, the development of a surface outpost also imposes additional requirements on the architecture, which will inevitably increase those costs as well. Capabilities such as pressurized rovers, unloading systems, and science labs were not included in the estimates made by Price et al.

If a progressive approach to Mars surface missions is pursued, with some period of short duration missions preceding any long duration activities, a cost profile can be achieved that is evenly loaded and therefore more likely to be sustainable. In NASA's anticipated exploration timeline, the bulk of the FY17\$10.9B of development cost for the inspace transportation capabilities would be expended in the seven year period between 2019 and 2026, supporting the anticipated launch of the DST in 2027 and the crewed Mars orbital mission starting in 2033. This represents an average annual base DDT\&E expenditure of FY17\$1.6B.

The FY17\$17.4B for the development of the surface access and ascent would then be expended in the seven year period between 2026 and 2033. The first lander elements would be launched in the mid-2030 timeframe to support the 2037 surface mission. This represents an average DDT\&E cost over the development period of FY17\$2.5B per year.

In order to achieve a more sustainable profile it is highly desirable to defer the development of the long duration surface capabilities until after the development of the short duration capabilities. In this scenario, the development of long duration capabilities would be deferred by a minimum of seven years, not starting until after 2033. Doing so would avoid overlaps between the DDT\&E for surface access and long duration and reduce funding peaks. Such a schedule would allow for a transition to long duration missions, if desired, for the 2043 or 2045 departure opportunities. If development of long duration capabilities are deferred, with no overlap with the short duration development, then the FY17\$14.6B would be expended over a seven-year period beginning sometime after 2033. The average annual DDT\&E expenditure in this period would be FY17\$2.1B.

However, if long duration missions are selected as the initial approach to human exploration of the Mars surface, then the full set of surface access and long duration surface infrastructure capabilities must be developed essentially simultaneously in the period between 2026 and 2033. The total DDT\&E cost of the minimal set of capabilities to enable long duration surface mission is FY17\$32.0B. This would result in an average annual base DDT\&E expenditure of FY17\$4.6B over this period. This amount represents a large spike in projected DDT\&E spending and is likely to be unaffordable under any projected budget profile, even ones that include a moderate increase in NASA funding. 
Cost is not the only factor that is related to the DDT\&E effort. There is also programmatic risk associated with any new capability development. Any time that a new critical path capability is added to the system, the risk of delay and/or cost overrun increases. Adding the long surface stay capabilities, in addition to the short surface stay, to the set of developments that must occur to enable the first human surface mission will increase the probability of programmatic delay.

The potential for short duration surface missions to allow for a more affordable campaign is a point that is emphasized in Lockheed Martin's Mars Base Camp architectural proposal. Lockheed Martin emphasizes that short surface stay missions are not just "flags and footprints" but rather enable the exploration of Mars on a near-term timescale providing a realistic, achievable architecture in the 2030s [5].

\section{Operational Cost \& Flexibility}

Capability development costs represents just a portion of the total resources dedicated to the human Mars campaign. Operational costs are also a significant contributor to the overall budget. Operational costs are particularly significant because they are not one-time costs, as with DDT\&E, but rather are recurring over the length of the program. For this reason operational costs directly impact the sustainability of the program.

The number of landers required to support each crewed mission is a primary discriminator between short duration and long duration missions. Additional landers must not only be acquired but also require additional in-space propulsion elements to deliver them to Mars, additional propellant, and additional launch vehicles.
As part of the EMC analysis, Goodliff et al (2016) [16] evaluated the number of landers required to enable a series of short duration and a series of long duration missions to the Martian surface, as seen in Figure VI.1. A number of different architectural solutions were evaluated in EMC with landers ranging in capacity from $18 \mathrm{t}$ to $27 \mathrm{t}$ cargo delivered to the Mars surface.

In the analysis presented by Goodliff et al, the long duration, single site mission requires four 20t landers (with LOX-Methane engines) to enable the initial human surface mission and 3 landers for each following mission. Although it should be noted that additional landers would eventually be required over time, as elements need to be replaced. Each lander requires an in-space propulsion stage, propellant, and SLS launch vehicles to launch the elements into space. The net result is that the initial long duration mission requires 14 SLS launches to complete the mission, with three crewed launches and 11 cargo launches. Each subsequent crew mission requires either 8 or 9 SLS launches to complete, with three crewed launches and either five or six cargo launches. The number of launches for subsequent missions varies because of the accumulation of propellant to refuel the in-space stages. The number of required SLS is reduced for subsequent missions because of the lower number of landers and because in-space propulsion stages are reused in the EMC architecture.

For a short duration mission, Goodliff et al estimate that two 24t landers (with storable propellant engines) would be required. This is likely a conservative estimate. Other architectures have presented solutions that utilize a single lander of similar capacity to enable short duration missions. However, these architectures typically involve other inspace capabilities to supplement the lander/ascent stage capabilities. Price et al (2016) [17] utilized a single 28t

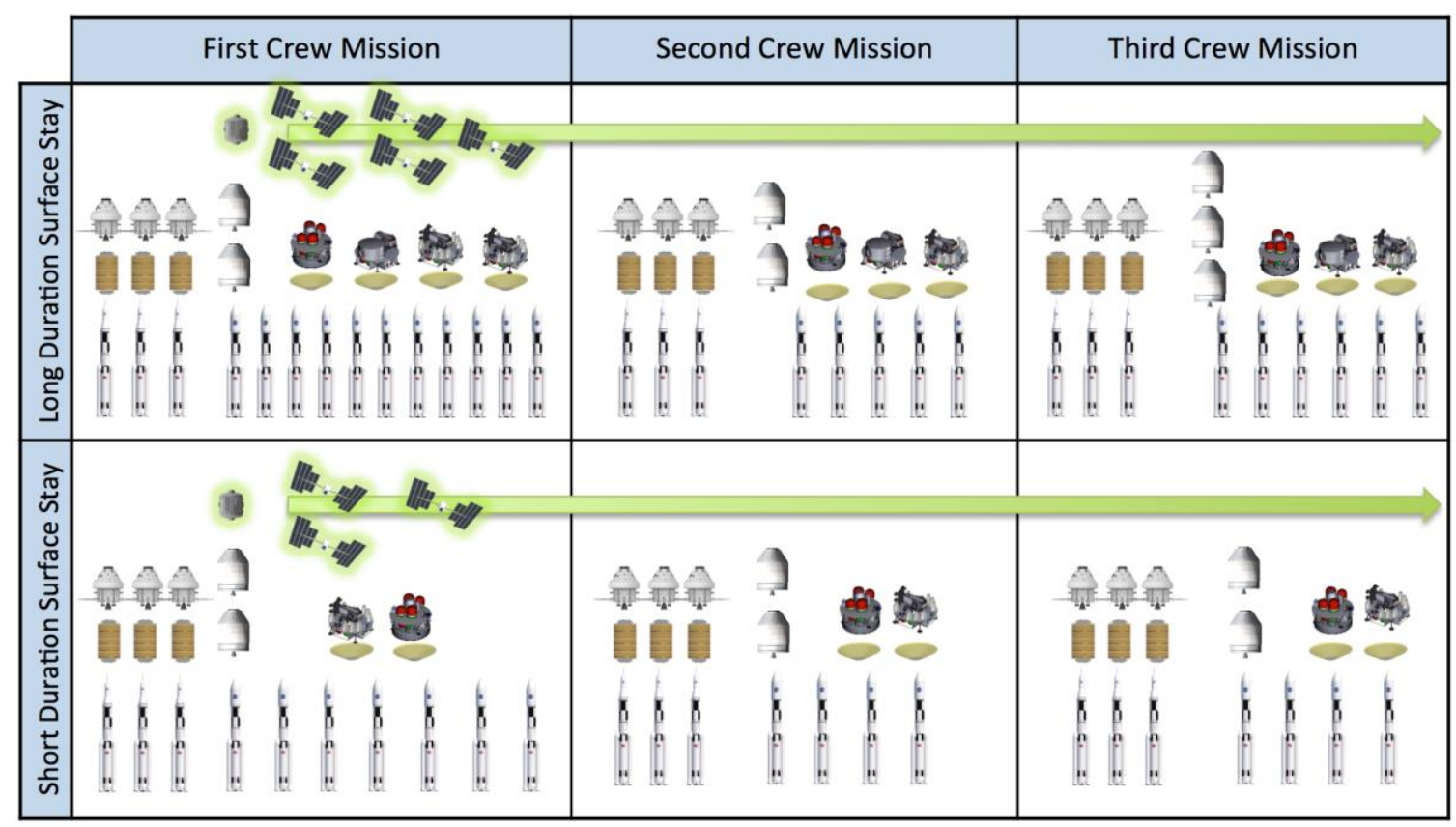

Figure VI.1 - Elements Required for Long Duration and Short Duration Surface Missions 
lander to enable short duration missions. However, this architecture also relied on a taxi vehicle to return the crew from low Mars orbit to the DST. As part of their Base Camp architecture [5] Lockheed Martin has proposed the use of a single lander that relies on refueling in Mars orbit. For the purposes of this comparison, two landers are assumed for the short duration mission.

Under these assumptions, the initial short duration mission requires a total of 10 SLS launches, with 3 crewed and seven cargo SLS launches. Each additional short duration mission requires a total of seven SLS launches, three crewed and four cargo. Again, the reduction in the SLS requirement is due to the reuse of in-space stages.

The difference in SLS launches between the short duration and long duration missions is particularly significant to the long-term sustainability of the Mars campaign. NASA projects that it has the capability to launch an average of two SLS per year, with a goal that at least one be a crewed launch. The current assumption is that a human mission to Mars would be conducted at every other Mars orbital departure opportunity, or approximately every 51 months. Conducting a mission at every opportunity would be too much of a strain on resources. Conducting missions less frequently would create large gaps in the program.

With a 51-month launch period, equating to the 51 months between crewed missions, there is an opportunity for approximately 8 SLS launches, four of which would be crewed and four cargo launches. The long duration mission requirement for 14 initial launches and 8 or 9 subsequent launches for each mission will stress the established SLS average flight rate. For the initial mission, launches will have to spread out over a number of years prior to the crewed surface mission. Since the orbital mission will also be conducted during this timeframe, the stress on SLS launches becomes even more severe. NASA currently anticipates having to launch the first SLS to support the 2037 crewed mission as early as 2034. Assets launched on these initial flights will have to loiter in space for very long periods of time prior to the crew launch. In addition, these early launches will also accelerate the development and acquisition of the cargo elements manifested on these flights.

Follow-on long duration missions will also stress the SLS launch rate. Launching five or six cargo missions in the 51month launch period will require either additional SLS launches and/or will require that some planned crew missions be converted to cargo missions.

In contrast, the short duration missions can more easily be accommodated under the projected SLS launch constraints. With only three crew and four cargo launches required, the launch campaign can be completed in the 51 month period with one additional SLS available to use for other missions.

The number of landers, stages, and launch vehicles required also directly impacts the on-going operational costs of the
Mars campaign. Acquiring the additional elements required to enable long-term missions will add significantly to the operational costs for the Mars program.

Flexibility in on-going operations is another potential advantage of the short duration, multiple site surface approach. Rather than being tied to a fixed outpost with large logistics demands, the short duration architecture can be re-planned and redirected over time. Lockheed Martin emphasizes this conclusion in their Mars Base camp architecture, noting that mission locations and operations can be tailored as new discoveries are made and as resources and areas of interest are identified.

In addition, in order to achieve the goals of pioneering and expanded human presence, the proposed long duration campaigns anticipate continued missions over an indefinite period. In effect, NASA could be "locked into" supporting these missions for a considerable period of time, largely eliminating the opportunity to pursue other exploration activities. A possible advantage of the short duration approach is that the specific goals of this phase can be achieved within an established timeframe, allowing NASA to move onto the next phase in exploration once completed.

\section{INITIAL SHORT DURATION MisSIONS}

The selection of surface mission architecture does not have to be a binary choice between short duration and long duration surface missions. There is a continuum of surface architectures with differing durations and capabilities that exist between the very short, Apollo-type missions and the full duration, full outpost type missions. Capabilities can be added and durations extended at one or multiple sites as budget and risk mitigation allows.

In addition, if pioneering-type goals continue to be important to NASA, it is always possible to transition from short duration to long duration surface missions at any point in the future. In the assumed approach, there is nothing about either the in-space architecture or the lander/ascent architecture that is unique to either mission. Once the capabilities to deliver the crew to Mars have been developed, the operational risks are reduced, sites are surveyed, and the hardware is proven, there is always an opportunity to transition to long-duration missions, if desired. As discussed in Section V, deferring long surface duration capabilities produces a budget profile that is more affordable and more sustainable. In addition, this approach also allows NASA to select an outpost site that has proven resources and is of particular scientific interest. Finally, a progressive development of surface capabilities could significantly reduce operational risk as the surface conditions and the impact on exploration systems are better defined.

\section{Summary}

A significant topic of concern among the mission architects, decision-makers, space enthusiasts, and other stakeholders 
in human Mars exploration, relates to the sustainability of the Mars campaign. For many of these stakeholders, the desire to conduct an infrastructure rich Mars campaign, including a 'Mars Outpost' and near-permanent human occupancy stems, at least in part, from the Apollo experience.

The argument that is advanced is that a repeat of the Apollo approach, pejoratively referred to as 'Flags and Footprints' does not provide adequate (or at least optimal) return on investment, will not result in a sufficient level of support from the public and from Congress, and that ultimately will lead to a dead-end in human Mars exploration.

This argument, however, assumes that it was the content of the Apollo missions that most directly contributed to the cancellation of the program; that, ultimately, the apparent value of the program did not justify further continued expenditures. However, this argument greatly oversimplifies why the Apollo program ended. While there was undoubtedly a decrease in the level of immediate public attention as the program progressed, that decrease is relative to the massive level of interest in the Apollo 11 mission. The public was still avidly watching each and every mission.

Similarly, the described lack of content in Apollo missions is also misleading. During the 600 total man-hours that astronauts spent on the Moon they explored six geologically different areas, collected and returned nearly $400 \mathrm{~kg}$ of samples, conducted 160 hours of crew-EVAs, deployed 26 science packages, and drove $90 \mathrm{~km}$ across the surface [18]. Data and samples from the missions are still being evaluated today, 45 years after the missions [19].

Rather, the primary driver in the cancellation of Apollo was the huge cost of ongoing operations. Even after DDT\&E was complete, the average annual ongoing operational cost of Apollo from 1969 to 1971 was approximately \$1.54B per year in 1970 ( $\$ 9.72 \mathrm{~B}$ in 2017 dollars), to conduct an average of 1.5 missions per year [20]. This represented $52 \%$ of the total NASA budget over that period. This was simply not a sustainable level of spending. Other priorities for NASA and for the nation would not allow for this continued level of investment, no matter what was being accomplished on the lunar surface.

This presents a valuable lesson for Mars exploration.

Controlling costs is a paramount factor in sustaining a program. Continued human exploration of Mars, if desired, can only be achieved through a program that has an affordable, sustainable budget profile. Achieving such a profile will likely require a carefully crafted, progressive approach to developing and deploying new capabilities. It is likely that NASA, even with help from international partners, will not be able to simultaneously develop and deploy all of the capabilities to deliver humans to the Martian surface AND the capabilities to survive on the surface for a year at a time.
In addition, the risk of Mars exploration is substantial and must be addressed in a progressive manner.

There should be no realistic fear that any human Mars program will suffer from a lack of public interest. Overall interest in all space activities and Mars activities are extremely high. There are now more ways for the public to follow and interact with missions than ever before, including social media and interactive VR technology that foster public participation in a Mars exploration experience. There is no lack of public interest in the ISS and Mars robotic programs. Surveys continually show a very high level of interest in space and in Mars missions in particular.

This is a case in which the over ambitious program is less likely to be sustained (or to ever be executed in the first place). Only by scaling our ambitions can we achieve an affordable and sustainable exploration program. As NASA moves forward in evaluating the architectural trade space for human Mars missions, it will be critical to include the analysis of short duration missions, which could result in satisfaction of most of the established goals and objectives, could reduce mission and crew risk, and may present the only affordable solution in the near-term. 


\section{REFERENCES}

[1] "Lunar Module Derivatives for Future Space Missions", The Grumman Corporation, 1965.

[2] Simberg, R., "Ending Apollo to Mars", Future In-Space Operations (FISO) Teleconference, 2016.

[3] "Human Exploration of Mars, Design Reference Architecture 5.0”, NASA-SP-2009-566, July 2009.

[4] Craig, D., Herrmann, N., and Troutman, P., "The Evolvable Mars Campaign - Study Status", IEEE Aerospace Conference, Big Sky, MT, 2015.

[5] Cichan, T., O’Dell, S., Richey, D., Bailey, S. and Burch, A., "Mars Base Camp Updates and New Concepts", 68h International Astronautical Conference (IAC), Adelaide, Australia, 2017.

[6] "Mars Exploration Zones", NASA.gov Video, 2015.

[7] "Mars Science Goals, Objectives, Investigations, and Priorities", Mars Exploration Program Analysis Group, 2010.

[8] Toups, L. and Hoffman, S., "Pioneering Objectives and Activities on the Surface of Mars", AIAA SPACE 2015 Conference and Exposition, Pasadena, CA, 2015.

[9] Human Exploration of Mars, Design Reference Architecture 5.0, Addendum", NASA-SP-2009-566-ADD

[10] Hoffman, S., "Evolvable Mars Campaign Development", NSF Large Facility Workshop, Smithsonian's S. Dillon Ripley Center, Washington, D.C., 2016.

[11] "Safe on Mars: Precursor Measurements Necessary to Support Human Operations on the Martian Surface", Committee on Precursor Measurements Necessary to Support Human Operations on the Surface of Mars, National Research Council, National Academies Press, Washington, D.C. 2002.

[12] Ming, D. and Morris, R., "Chemical, Mineralogical, and Physical Properties of Martian Dust and Soil", Astromaterials Research and Exploration Science Division, NASA Johnson Space Center, Houston, TX, 2017.

[13] Price, H., Hawkins, A. and Radcliffe, T., "Austere Human Missions to Mars", AIAA SPACE 2009 Conference and Exposition, Pasadena, CA, 2009.

[14] "Pathways to Exploration: Rationales and Approaches for a U.S. Program of Human Space Exploration", National Research Council, The National Academies Press, Washington, D.C., 2014.

[15] NASA's Plans for Human Exploration Beyond Low Earth Orbit", NASA Office of Inspector General, NASA IG-17-017, Washington, D.C., 2017.
[16] Goodliff, K., Troutman, P., Craig, D., Caram, J. and Hermann, N., "Evolvable Mars Campaign 2016 - A Campaign Perspective", AIAA SPACE 2016 Conference and Exposition, Long Beach, CA, 2016.

[17] Price, H., Baker, J., and Naderi, F., "A Minimal Architecture for Human Missions to Mars - An Update", Future In-Space Operations (FISO) Teleconference, 2016.

[18] "The Apollo Program", Smithsonian National Air and Space Museum, www.airandspace.si.edu/explore-andlearn/topics/apollo/apollo-program/orbital-missions.

[19] "Lunar Rocks and Soils from Apollo Missions", Curation Lunar, National Aeronautics and Space Administration, Washington, D.C. 2016.

[20] "The Apollo Spacecraft: A Chronology", vols. 104, NASA SP-4009, Washington, D.C., 1969-1978.

\section{BIOGRAPHY}

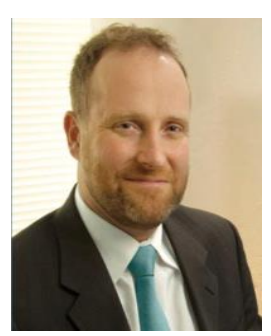

Chel Stromgren currently serves as the Chief Scientist of Binera, Inc. Risk Analytics Division. In this role, Mr. Stromgren leads the development of probability and riskbased strategic models and strategic analysis of complex system development. Mr. Stromgren has supported NASA in the analysis of Space Shuttle and International Space Station operations in the post-Columbia environment and has led the development of strategic campaign models for the Mars exploration initiatives. He holds a Bachelor of Science degree in Marine Engineering and Naval Architecture from the Webb Institute and a Master of Science degree in Systems Management from the Massachusetts Institute of Technology.

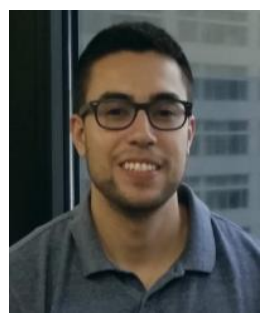

Felipe Escobar currently serves as a Senior Risk Analyst at Binera, Inc., where he has worked for over five years in the areas of complex systems analysis and probabilistic modeling. In this role, Mr. Escobar supports the development and analysis of space systems and campaign planning models for NASA's Human Space Flight studies. He received a bachelor's degree in International Economics from Boston University in 2011.

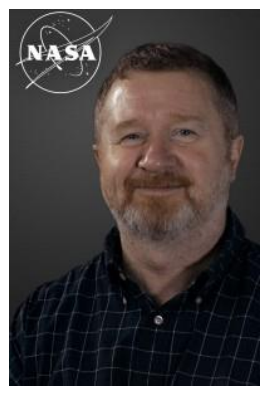

William Cirillo currently serves as a Senior Researcher at NASA Langley Research Center in Hampton, Virginia, where he has worked for the past 20 years in the area of Human Space Flight Systems Analysis. This has included studies of Space Shuttle, International Space Station, and Human Exploration beyond low Earth orbit. In 2005, Mr. Cirillo served at NASA Headquarters as a core member of the Exploration Systems Architecture Study (ESAS) team, 
where he was responsible for studying the use of Ares I/Orion in meeting future ISS crew and logistics transportation needs. Mr. Cirillo currently leads a team of analysts in assessing at a strategic and tactical level the manifesting of assembly and logistics flights human exploration beyond low Earth orbit.

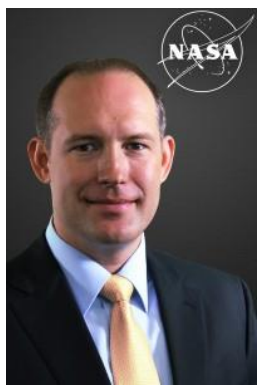

Raymond (Gabe) Merrill currently serves as the lead of the Mars inspace transportation team for Human Exploration and Operations Mission Directorate (HEOMD) Mars Study Capability (MSC). He earned a B.S. in Aerospace Engineering from North Carolina State University in 2001. Mr. Merrill began his career at NASA Langley developing, integrating, and running systems and trajectory analysis for reusable launch vehicle system technology assessments, Constellation lunar mission formulation, and Revolutionary Aerospace Systems Concepts (RASC) Mars mission concepts. Over the past 15 years he has been a part of various human spaceflight architecture teams specializing in power, propulsion, and mission design concepts to support exploration beyond low Earth orbit.

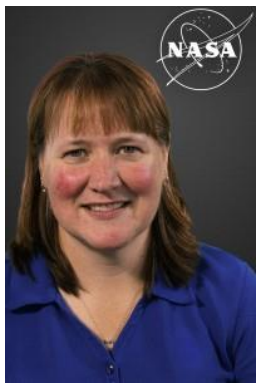

Kandyce Goodliff is an aerospace engineer at NASA Langley Research Center in Hampton, VA, with the Space Mission Analysis Branch (SMAB). Her primary roles as a systems analyst for SMAB are conceptual design and sizing of human and robotic spacecraft, mission and spacecraft analysis, and campaign analysis for human exploration. She has a Bachelor of Science in Aerospace Engineering from Embry-Riddle Aeronautical University and a Master of Science in Mechanical Engineering from the George Washington University. 